\title{
Fostering communication and collaboration through regenerative design games.
}

\author{
Niki Wallace \\ University of the Arts London
}

\begin{abstract}
:
This paper explores how regenerative design games might foster the communication and collaboration skills needed to scaffold transitions to just and sustainable futures. It explores three design games, each co-designed with different people, for different intents and purposes but each with the shared aim to build people's capacity for regeneration, open communication and effective collaboration. It discusses the role of games in explorations of uncertain futures and highlights the important role played by care practices in co-design processes.
\end{abstract}

Keywords: design for transitions; design games; communication; collaboration; microsolidarity

\section{Introduction}

Transitioning toward futures that are socially just and ecologically sustainable will likely involve complex design engagements that are global, multi-disciplinary, and cross-sectoral. (Boehnert, 2018; Ceschin \& Gaziulusoy, 2016; Irwin, Tonkinwise, \& Kossoff, 2015). Designers' capacity to engage with plural perspectives and work across such dynamically shifting contexts will affect their contributions to these teams, and more broadly, to transitions (N. Bateson, 2016; Escobar, 2018; Fry, 2020).

Operating effectively in these teams requires advanced practice of 'soft skills' such as communication and collaboration. The transcontextual nature of these engagements affects how and where seeing happens, by whom and in what context. Practicing design for transitions might also demand Batesonian practices such as transcontextual seeing and symmathesy (mutual learning) to support collaborations (G. Bateson, 1979; N. Bateson, 2016).

This paper presents three regenerative design games that foster communication and collaboration. It explores how the co-design process for such games can build designers' capacity to design for transitions, how participation in gameplay can build players' capacity to participate in societal transitions, and how both can foster regenerative cultures. 


\section{Background}

\subsection{Co-design}

Co-design has its roots in the Scandinavian practice of participatory design where participants are partners in generative design processes, however the term co-design also describes a more extractive process of seeking feedback from selected end-users of a product or service (Bjögvinsson, Ehn, \& Hillgren, 2012; E. B.-N. Sanders \& Stappers, 2008). Co-design (with generative intent) has been described by Sanders and Stappers (2012) as a tool, technique, method and mindset, and has commonly centred the co-design workshop as the time and space for participation. We are now seeing shifts in the ways in which participation occurs in response to COVID and social distancing requirements. The author has recently contributed to the development of 'low-contact co-design' (Davis et al., 2021) which proposes blended assemblages of different tools and techniques and offers improvements for inclusivity through generative processes that amplify participation. The design games presented in this paper have been co-designed with generative intent using the low-contact co-design approach (Davis et al., 2021).

\subsection{Regenerative cultures}

In its simplest definition the act of regeneration means 'to create again' and in the context of this research, regenerative cultures are a desirable cultural outcome of participation. Deeper understandings of regeneration might also be built through explorations of its tensions; as a restorative feedback loop that is capable of being exploited, capitalised and colonised in some contexts and held, nurtured and shared in others. The author has witnessed through their work in an Australian peri-urban community how regenerative agriculture practices are improving soil health whilst also recolonising unceded Aboriginal land. Similar tensions are seen in a rewilding context in the UK, which though regenerative on face value, is also pre-loaded with colonial understandings of 'wilderness' and 'wildness' that erase the long histories of Indigenous relations with land (Plumwood, 2002; Ward, 2019). Lee (2005) describes how the rooting of Kaupapa Māori theory and the pūrakāu method for Māori narratives in academic settings in Aotearoa is nurturing the regeneration of Māori cultural pedagogies, though not fully seeded, this gives a glimpse at possible regenerative futures.

The language and concepts of regeneration have been taken up in many design contexts, few have more ramifications than the built environment, which impacts (and often destroys) a living system through the realisation of a built system. Cole, Oliver, and Robinson (2013) describe how "a regenerative approach [requires] the development of strategies to deal with uncertainty, particularly those emerging from the fact that the human systems in the building are endowed with volition and intentionality" (2013, p 239). This recognises the roles of people and place in regeneration and the ways both add uncertainty that can lead to unintended consequences in design outcomes.

A sensitivity to these tensions and the binds they present has informed the co-design of the games presented here. This has facilitated the players' participation in mutual learning processes that developed and broadened their own perspectives though embodied regenerative experiences. Empathy building across human and more-than-human perspectives was a key aim for cultural regeneration. Each game seeks to renew and restore through acts of care and participation rather than colonise and conquer through competitive forces. 


\section{Methodology and Methods}

This research is framed by critical pragmatism (Forester, 2013), which is a deliberative, critical, participatory, action oriented perspective. Critical pragmatism is attentive to the invitation to participate and the possible futures that participation affords. Informing this view are plural, decolonial, political perspectives from Escobar (2018), Fry (2020), Esteva (1987), Plumwood (2002) and Yunkaporta (2019). Each game used low-contact co-design methods (Davis et al., 2021) and rapid prototyping to co-create multiple games with the aim to build players' communicative capacity and to encourage collaboration through gameplay. Reflective practice (Schön, 1983) and the author's reflective doodling method (Wallace, 2020) have informed an iterative process of embodied reflection throughout the co-design process and beyond.

The three games presented in this paper were each played with test groups from the respective codesign teams' social networks, followed by game play with the public. Feedback was provided by players from both test groups and the general public through an open invitation for post-game reflection and generative discussion. These conversations informed the reflective and iterative codesign approach taken for these games and provided insights on the regenerative impact of participation in play.

Each co-design process was scaffolded by the practice of microsolidarity (Bartlett, 2018), which provided mutual aid through care and support in small group settings. Microsolidarity gave each team a set of practices through which they could care for one another while working with one another. This was a key aspect of the approach for three reasons: firstly, it recognised that care in working relationships happens whether formalised or not but will typically fall on women as a form of gendered labour (Serrano-Pascual, Artiaga-Leiras, \& Crespo, 2019). To counter this, care labour and practices were consciously distributed throughout the team. Secondly, it recognised that each team member was performing labour in a pandemic that created unprecedented working conditions; this demanded deeper care and support. Thirdly, each team was co-designing a game that touched upon serious, complex and systemic problems that pose an existential threat. The nature of this work comes with a significant cognitive load; microsolidarity practices provided each team with the time and space needed to unload. Each team found solace through their practice of microsolidarity and although what emerged through its practices was unique to each team, it was consistent in providing care and mutual aid.

\section{Regenerative Design games}

These findings reflect on the regenerative and restorative act of the co-design process and gameplay in three design games, Better than Before, Food Futures and More-than-human. The games were codesigned purposefully to enable, practice and nurture communication and collaboration skills, and to build people's capacity for, acceptance of, and participation in societal transitions.

\subsection{Better than Before}

In the conversational card game, Better than Before, a simple set of cards was used to structure challenging conversations about post-pandemic futures within households. Its educational objectives and heavy subject matter perhaps make 'serious game' (Peng, Lee, \& Heeter, 2010) a better description for it. Better than Before was co-designed in lockdown, for lockdown, by the author, Climate Space (a team of climate action facilitators and designers), and the CE of their local peak body for conservation. The game consisted of three rounds of questions, designed to help people to probe their experience of the pandemic to uncover what was now visible, what felt possible and 
what remained challenging for them. The overarching aim was to hold space for atypical conversations within households or family units and open up challenging conversations through a safe process that allowed people to navigate their fears while exploring their hopes. The card set was designed for self-facilitation with carefully crafted questions that helped guide people through these challenging but safe conversations (see Figure 1).

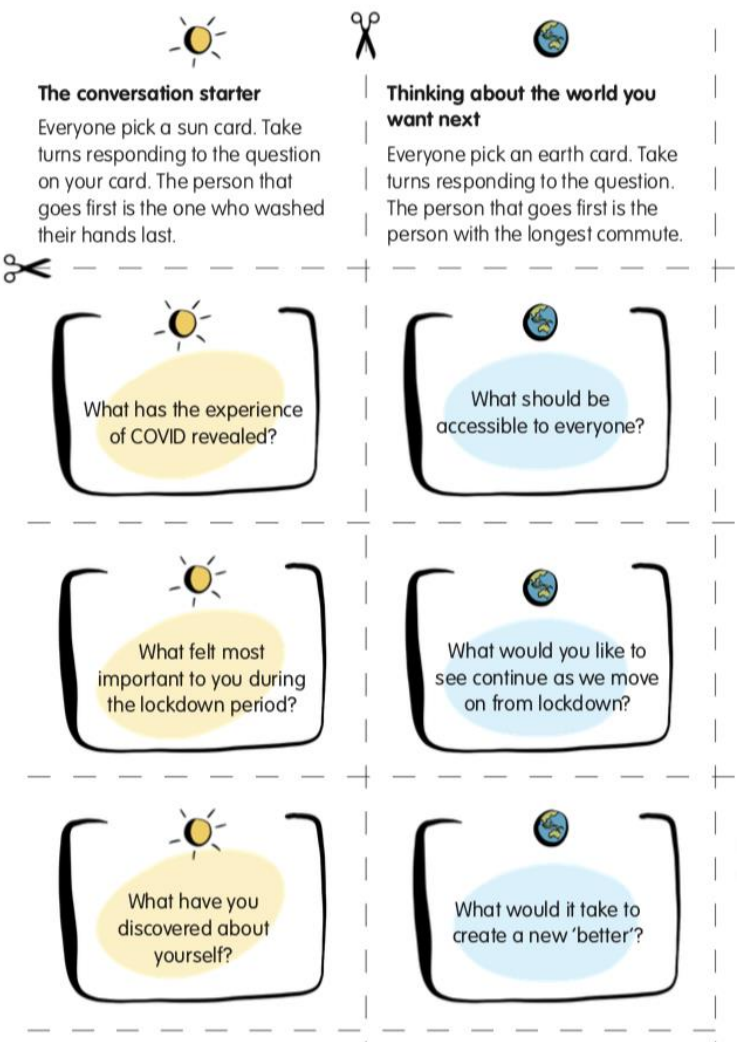

Figure 1. Better than Before sample question cards.

Better than Before was a proposition for participatory democracy and invited players to 'do something' after their conversation. An online tool facilitated sharing and voting on ideas with the intention to feed the most popular ideas through to local parliament. Ideas such as universal basic income and electric vehicle transitions were popular, as was the idea to "celebrate people looking after one another and their community". The leading idea, "for governments to take brave and extensive action to enforce some changes needed to reduce climate change", was upvoted by $86 \%$ of participants suggesting a strong and urgent desire for climate action in the political sphere.

Beyond the aim to encourage open conversations about difficult circumstances, the game also aimed to tie hope to action by encouraging the publics' critical reflection and active participation in the crafting of possible futures. It extended their democratic participation beyond electoral voting to provide spaces where more voices might be heard. The team's experiments with gameplay highlighted the crucial role played by the phrasing of the questions and how important 'warming up' the space is when inviting deep communication. Better than Before also revealed the importance of deep listening and holding space for challenging conversations amidst crisis.

\subsection{Food Futures}

Food Futures is a role play card game (also played online) designed to engage members of a periurban community and explore vulnerabilities in their local food system. It was co-designed in the 
author's living lab by designers, researchers, and community members as part of a larger complex design engagement. Role playing offers a unique separation from the self that can build empathy for the experiences of others by 'playing them' socially and reimagining the world through their eyes. In Food Futures, players select a character at random and through the gameplay acquire different skills and roles which they can harness in their response to an unfolding crisis in their community (see Figure 2). Players are invited to add themselves to the card deck at the end of each game with the intent of generating a more bespoke card set that is reflective of real community members.

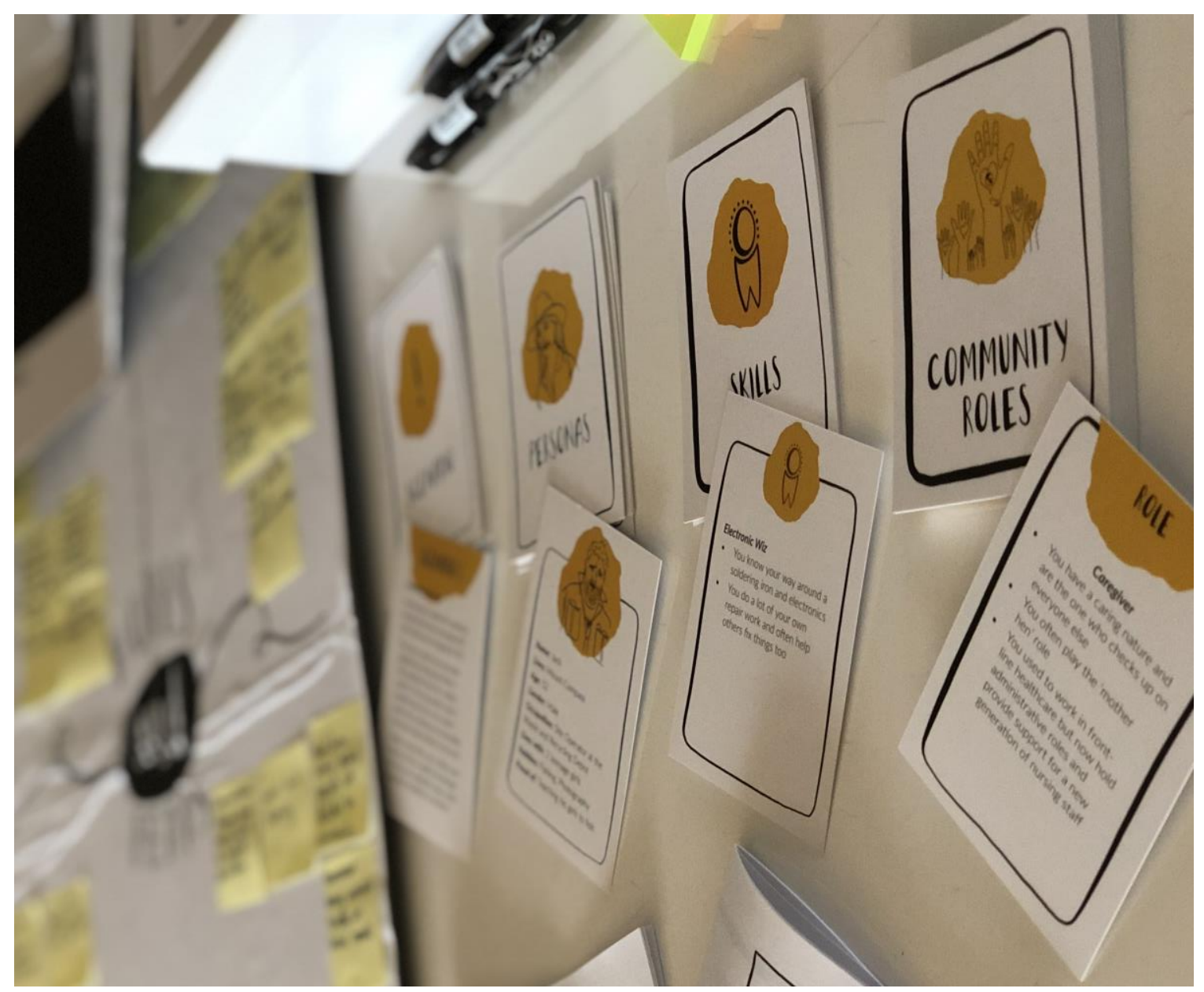

Figure 2. Prototype card set for Food Futures.

In different iterations of the gameplay the co-design team explored how players might respond with or without leadership/directives. Of interest here was the way some players began working together more closely in the absence of leadership directives, while for others, the lack of direction became a barrier to imagining possibilities for action. The combination of empathy through role play and adaption through crisis response aimed to build the adaptive capacity of players. When reflecting on the 'retrospectives' it became clear that the game provided something different to each player. For community members ready to collaborate it provided a playground for practice; their imaginative and participatory responses became open invitations for fellow players to collaborate. For those unable to 'see' the food system it provided a way in; one participant noted that "I didn't really know what the food system was but when my group discussed the food shortages during the pandemic I got it, I could see it". For the co-design team it built capacity in transcontextual seeing, prompting deeper consideration of 'who else?', 'what else?', 'where else?' and 'how else?', fostering critical engagement with more plural perspectives. Over time, community members add their personas, roles and skills to the deck and this generative card set becomes highly representational of the 
community itself, thereby making it a valuable tool for future processes. This could also mean that for those in leadership roles, the card set has the potential to gamify crisis modelling processes by providing a bespoke tool for modelling with their particular community in mind.

\subsection{More-than-human}

More-than-human is an online game that explores the climate crisis through the perspectives of the flora and fauna from Papua New Guinea, one of the most biodiverse regions in the world (see Figure 3). The game is a collaboration between the author and a design strategist based in the Philippines. It emerged from their shared practice of microsolidarity as a form of 'microsolidarity-in-action', where creative collaboration is an outcome of their care practices rather than care practices being a scaffold for their work. More-than-human responds to the need for regenerative cultures and is being codesigned iteratively in response to each post-game discussion. The game's narrative has been carefully designed to take people through a safe but confronting experience of climate crisis whilst using the techniques of microsolidarity to scaffold the players' experiences.
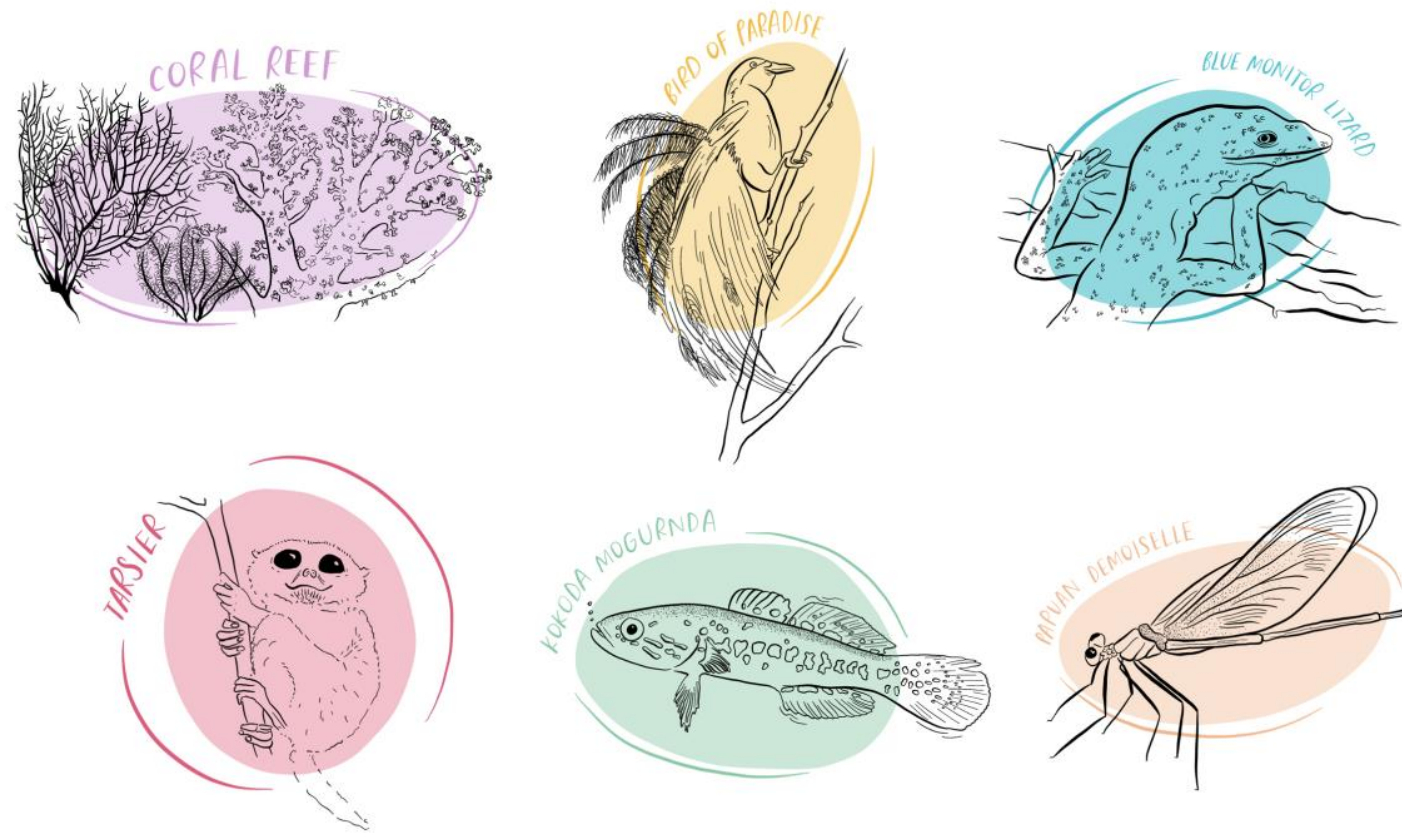

Figure 3. More-than-human characters.

More-than-human uses Zoom and Mural to provide an immersive online experience. A fully illustrated Mural board acts as a collaborative gameboard where storytelling, art, education, music, generative ideation and critical conversation come together to support gameplay that explores the climate crisis through the lens of each more-than-human character. The characters are played in pairs, less as a role-playing or 'acting' exercise and more as a cognitive, 'role-taking' process, which communicates a temporary imagining of a scenario from another's perspective rather than acting out the behaviours or mannerisms of another (Peng et al., 2010).

The communication and collaboration needed for gameplay is pre-primed through relational processes that are then interspersed throughout the game. This might enable the coral reef's collaboration with the insects or fish while the birds of paradise might attempt to use their beauty to influence the humans. As dangerous weather conditions and the unfolding climate crisis create continual twists and turns, adaptive responses are demanded of the characters. 
The game is intimate yet confronting, and participant feedback indicates a generative and powerful experience of empathy and plural perspectives emerges from gameplay. One participant described it as "a really powerful experience of empathy and perspective-brought home quite clearly the role of home-every living being has a place on this planet". This stretching of empathy was noted by several participants who expressed an appreciation for the challenge presented by thinking from more-than-human perspectives. Others described their emotional journey, with one participant sharing how "I went through a whole range of emotions, none of them too strong, but all noticeable. There was a moment of huge frustration, and I went into denial at some point, I thought there would be a happy ending-we'll survive this-but no, that's real life." More-than-human intentionally reaches crisis point and then unapologetically escalates it. Though the game invites participants to sit with their discomfort, it also scaffolds the experience through open conversations and a hint of fun.

\section{Discussion}

Design games have a long history in co-design, where card sets, role play and other games are often used as part of participatory design processes (L. Sanders \& Stappers, 2012). Gamification as a strategy has also been used in design, health, education and learning management (Bergen, Solberg, Sæthre, \& Divitini, 2018; De Jans, Van Geit, Cauberghe, Hudders, \& De Veirman, 2017; Jessen, Mirkovic, \& Ruland, 2018; Pirinen, 2016) and though it can be used commercially as an 'engagement hack', here gamification is better understood as a tool for enhancing regeneration through interaction and involvement. Gamification is also argued by McGonigal (2011) as a strategy for the development of 'future making' skills that are transferrable from gaming environments into the real world, where skills acquired through gameplay can be applied to real-world problems. This is evident in the numerous games designed by McGonigal, including World Without oil, EVOKE, and SuperStruct, each of which immerses players in games that develop their complex problem-solving abilities and prompt players' personal transformation in the process (McGonigal, 2011).

The social nature of the regenerative games discussed below is highly valuable as they can create engaging participant experiences whilst also offering benefits through the social processes of codesign. These games revealed how gamification can also be used with different kinds of intimate intentionality-to unlock communication, to invite collaboration and to foster regeneration.

\subsection{Communication and collaboration in the co-design process}

Each co-design process was unique and relational but each one also centred the relationships and nurtured them through structures for work and care; team members benefited in multiple ways from this approach. The relational processes each team engaged with appeared to enhance both the communication between team members as well as the team's capacity for collaboration. Beginning each design sprint by setting the sprint's purpose, rhythm and aims and the team members' roles and responsibilities created accountability agreements within the teams. Working iteratively meant that over time, teams also experienced a smoothing of their flow, felt as a kind of 'coming together' of people and processes. Social processes for checking in/out of co-working sessions allowed people to feel whole in the work they did whilst providing space for mutual support. Ending each cycle/game with a 'retrospective' held space for reflection with the design team and players of early prototypes. The openness of these communication processes allowed teams to work at their best, to accommodate and care for one another even when feeling at their worst, and to use co-design to enhance gameplay and improve the end-outcomes. At a meta level, though mostly tacit, this also allowed teams to practice some of the same communication and collaboration techniques that the games were attempting to nurture. 


\subsection{Microsolidarity and the role of care in collaboration}

One of the key reflections on each game was on the significant role played by microsolidarity in the co-design process. Although design sprints were used as a response to the time-critical nature of each project, it was microsolidarity that facilitated this pace rather than the design sprint structure. Microsolidarity's 'care pod' practice created a harmonious rhythm for care activities alongside the rhythm for work activities. Care pods fostered rapid development of high-trust relationships which in turn enabled more effective communication and collaboration. The use of these communication and collaboration practices in the co-design process is particularly interesting when considered in relation to Conway's Law, which describes how the communication structure of an organisation is reflected in the end outcome of that organisation's work (Kim, 2016). In light of this, it might be argued that microsolidarity has been imprinted in each of these games and through these trace elements, also provides players with micro-experiences of microsolidarity.

\subsection{Designing with familiar formats to explore unfamiliar territory}

Games that tackle crises such as the COVID pandemic and the climate crisis have a particular responsibility to players' psychological safety. How players are held and supported through the gameplay must be carefully considered by designers of such games. The discomfort of confronting crises, of taking them seriously while 'playing', likely positions most of these games as 'serious games', where the nature of the content and its aim to cultivate the cultural conditions for social change adds a gravitas to the gameplay (De Jans et al., 2017; Peng et al., 2010). In an effort to smooth any discomfort, the familiar and nostalgic formats of card games and board games were used in Better than before and Food Futures, for their provision of a familiar foundation on which to explore an unfamiliar crisis. Despite this intentional inclusion of the familiar, it is more likely the care provided by a game's host that aids players' ability to sit in discomfort and explore crisis. In some respects this is curating more than a 'safe space' where players feel 'held' in the process, it is also creating a 'brave space', where a player might require courage as their actions shift them outside of their comfort zone. This was most evident in more-than-human, which uses less conventional elements of gameplay and relies on behaviour modelling by the hosts who play the Tarsiers, the tinyprimate narrators and holders of the safe-brave space in which the game is played.

\subsection{Curating spaces and priming participation.}

Curating the space in which communication and collaboration occurs is key to each of the games presented here and was typically done in multiple ways, but initially through the design of each game. The visual language in each of these games offers a friendly invitation to play, by inviting people in with playful illustrations, line styles and colours that are paired with more formal elements that indicate to players, 'this is serious, but we can still have fun'. 'Warming up' the space (even online) is communicative, done in these games through the inclusion of music and stories.

Participation is primed through check in processes that invite 'relationshipping'; this enables collaboration by encouraging being whole and present to what is unfolding. Gathering online is becoming more familiar, as this familiarity grows, we can recognise where layers of communication have been stripped and where new ones are emerging. What can be read (neurotypically speaking) in the space during an in-person encounter is dramatically altered in online environments. While for some the shift to online gatherings has equalised participation, for others it has become significantly more challenging. Understanding and responding to this communicative challenge is a continuing aspect of this work. 


\subsection{Power and play}

In his discussions on power dynamics, Dowding (1996) uses game theory to describe how people will often cooperate within a game until this presents a disadvantage by hindering their potential to win. Although winning appears to be an inherent aspect of most games, notions of winning in regenerative design games can be altered to instead emphasise collaboration as a 'benefit for all' rather than playing to 'win for one'. More-than-human takes this further by integrating an alternative judgement of winning into the gameplay. To finish the game, players participate in a round of voting to determine which character gave the best responses, thereby choosing a winner collaboratively through social agreement processes. The 'winner' is announced as the new Secretary General of the UN, revealing that the power assigned to 'winning' comes with responsibility. Reflecting on postgame feedback from one player who noted the power dynamics between these characters in nature, is a reminder that power is ever-present in every system, and especially so in times of crisis. The inclusion of predators and prey and notions of home in more-than-human plays with this idea and invites players to engage with the tensions it brings.

\section{Conclusion}

This paper has presented three regenerative design games that were co-designed in response to multiple crises, set against the backdrop of climate crisis. Each game helps to enable regeneration, communication and collaboration in participants, and of note was how the co-design process of each game also enabled regenerative cultures within the co-design teams. The important roles played by co-design and particularly microsolidarity are key here, as both appear to bring benefits to the design team and the game's players. A game's format and its hosts are also key to how space is held and how it becomes occupied through communicative and collaborative engagement. The importance of curating that space, priming participation with 'relationshipping' processes, and being attentive to power in design and in gameplay are all key takeaways. Reflection reveals the important role each of these games play through the practice of transcontextual seeing, through effective communication and collaboration, and by fostering regenerative cultures. As these games continue to be played new prototypes are emerging, particularly for more-than-human, where upcoming prototypes include an urban edition with characters such as street cats, pigeons and cockroaches, and an edition designed more specifically for intergenerational gameplay. As this work continues, it aims to expand upon this initial exploration of the use of design games for regeneration, communication and collaboration and to observe the role they play in design for transitions. Of particular interest is the relation between design and ecopsychology, where the dynamic interplay between gamer/game, social/ecological, and process/play can be examined more intentionally and in greater depth than this paper permits.

\section{References}

Bartlett, R. D. (2018). Microsolidarity. Retrieved from https://www.microsolidarity.cc Bateson, G. (1979). Mind and nature: A necessary unity (Vol. 255): Bantam Books New York. Bateson, N. (2016). Small arcs of larger circles: Axminsters: Triarchy Press.

Bergen, E., Solberg, D. F., Sæthre, T. H., \& Divitini, M. (2018). Supporting the co-design of games for privacy awareness. Paper presented at the International Conference on Interactive Collaborative Learning.

Bjögvinsson, E., Ehn, P., \& Hillgren, P.-A. (2012). Design Things and Design Thinking: Contemporary Participatory Design Challenges. Design Issues, 28(3), 101-116.

Boehnert, J. (2018). Design, Ecology, Politics: Towards the Ecocene: Bloomsbury Publishing.

Ceschin, F., \& Gaziulusoy, I. (2016). Evolution of design for sustainability: From product design to design for system innovations and transitions. Design Studies, 47, 118-163. 
Cole, R. J., Oliver, A., \& Robinson, J. (2013). Regenerative design, socio-ecological systems and co-evolution. Building Research \& Information, 41(2), 237-247.

Davis, A., Wallace, N., Langley, J., Gwilt, I. (2021) Low-Contact Co-Design: Considering more flexible spatiotemporal models for the co-design workshop. Strategic Design Research Journal, 14(1).

De Jans, S., Van Geit, K., Cauberghe, V., Hudders, L., \& De Veirman, M. (2017). Using games to raise awareness: How to co-design serious mini-games? Computers \& Education, 110(1), 77-87.

Dowding, K. (1996). Power. Buckingham: Open University Press.

Escobar, A. (2018). Designs for the pluriverse: radical interdependence, autonomy, and the making of worlds: Duke University Press.

Esteva, G. (1987). Regenerating people's space. Alternatives, 12(1), 125-152.

Forester, J. (2013). On the theory and practice of critical pragmatism: Deliberative practice and creative negotiations. Planning Theory, 12(1), 5-22. doi:10.1177/1473095212448750

Fry, T. (2020). Defuturing: A new design philosophy. London: Bloomsbury.

Irwin, T., Tonkinwise, C., \& Kossoff, G. (2015). Transition design: An educational framework for advancing the study and design of sustainable transitions. Paper presented at the 6th International Sustainability Transitions Conference, University of Sussex, Brighton.

Jessen, S., Mirkovic, J., \& Ruland, C. M. (2018). Creating Gameful design in mHealth: a participatory Co-Design approach. JMIR mHealth and uHealth, 6(12), e11579.

Kim, G. (2016). How to Design With Conway's Law in Mind. Retrieved from https://dzone.com/articles/how-todesign-with-conways-law-in-mind

Lee, J. (2005). Mäori cultural regeneration: Püräkau as pedagogy. Paper presented at the 3rd International CRLL Conference: What a difference a pedagogy makes: Researching lifelong learning and teaching, Stirling, Scotland.

McGonigal, J. (2011). Reality is broken: Why games make us better and how they can change the world. Penguin.

Peng, W., Lee, M., \& Heeter, C. (2010). The effects of a serious game on role-taking and willingness to help. Journal of Communication, 60(4), 723-742.

Pirinen, A. (2016). The barriers and enablers of co-design for services. International Journal of Design, 10(3), 2742.

Plumwood, V. (2002). Environmental Culture: the ecological crisis of reason. London: Routledge.

Sanders, E. B.-N., \& Stappers, P. J. (2008). Co-Creation and the New Landscapes of Design. CoDesign, 4(1), 5-18. doi:10.1080/15710880701875068

Sanders, L., \& Stappers, P. J. (2012). Convivial Toolbox: Generative Research for the Front End of Design. Amsterdam: BIS Publishers.

Schön, D. A. (1983). Reflective practitioner: how professionals think in action. New York: Basic Books.

Serrano-Pascual, A., Artiaga-Leiras, A., \& Crespo, E. (2019). The gender of care: Repertoires of Emotion Regulation and the Moral Foundations of Micro-solidarity. Revista Española de Investigaciones Sociológicas, 166, 153-168.

Wallace, N. (2020). Thinking while drawing and drawing to think: Exploring 'reflective doodling' as a critical reflective practice in design for transitions. Proceedings of DRS 2020, 204.

Ward, K. (2019). For wilderness or wildness? Decolonising rewilding. In Rewilding (pp. 34): Cambridge University Press.

Yunkaporta, T. (2019). Sand talk: How Indigenous thinking can save the world: Text Publishing.

Author Bios:

Dr Niki Wallace is an academic and founder of a living lab that catalyses transitions in communities through co-research and co-creation. The lab's work complements Niki's academic research in design for transitions, circular economies, and co-design. Play with me on Twitter: @nikiwallace. 
Acknowledgements: this research was conducted whilst I lived and worked on Kaurna country. I acknowledge the Traditional Owners of country throughout Australia and recognise their continuing connection to land, waters, and culture. I pay my respects to Elders past and present. I acknowledge that regenerative cultures and the processes of relationality, embodied reflection and drawing that I frequently engage in are part of a rich tradition in Indigenous Cultures (particularly Aboriginal and South American). I wish to acknowledge the privilege that is tied to working with these cultural practices as a white Euro-Australian woman. I am eternally grateful for the significant influence Indigenous Knowledges have on how I think about and do my work. I would also like to thank my codesign partners from Net Zero Lab, THE GLOBAL ROOM, Climate Space, and Conservation $\mathrm{SA}$, they made these projects both possible and pleasurable. I'm also grateful to the peer reviewers of this paper, their kind and supportive remarks improved this paper. 